\title{
A MULTIDIMENSIONAL FLUCTUATION SPLITTING SCHEME FOR THE THREE DIMENSIONAL EULER EQUATIONS*
}

\author{
JÉRÔMe BASTIN ${ }^{1}$ AND GILBERT RogÉ ${ }^{2}$
}

\begin{abstract}
The fluctuation splitting schemes were introduced by Roe in the beginning of the 80's and have been then developed since then, essentially thanks to Deconinck. In this paper, the fluctuation splitting schemes formalism is recalled. Then, the hyperbolic/elliptic decomposition of the three dimensional Euler equations is presented. This decomposition leads to an acoustic subsystem and two scalar advection equations, one of them being the entropy advection. Thanks to this decomposition, the two scalar equations are treated with the well known PSI scalar fluctuation splitting scheme, and the acoustic subsystem is treated with the Lax Wendroff matrix fluctuation splitting scheme. An additional viscous term is introduced in order to reduce the oscillatory behavior of the Lax Wendroff scheme. An implicit form leads to a robust scheme which enables computations over a large range of Mach number. This fluctuation splitting scheme, called the Lax Wendroff - PSI scheme, produces little spurious entropy, thus leading to accurate drag predictions. Numerical results obtained with this Lax Wendroff PSI scheme are presented and compared to a reference Euler code, based on a Lax Wendroff scheme.

Résumé. Les schémas distributifs sont apparus au début des années 80 grâce à Roe et ont connu de nombreux développements jusqu'à aujourd'hui, notamment sous l'impulsion de Deconinck. Dans cet article, nous rappelons le formalisme des schémas distributifs avant de présenter la décomposition hyperbolique elliptique des équations d'Euler tridimensionnelles. Cette décomposition permet d'obtenir un sous-système acoustique et deux équations d'advection scalaire, dont l'advection de l'entropie. Grâce à cette décomposition, les deux équations scalaires sont traitées par le schéma PSI, célèbre schéma distributif scalaire, et le sous-système acoustique est traité à l'aide du schéma distributif Lax Wendroff matriciel. Une viscosité artificielle est utilisée pour réduire le caractère oscillant du schéma de Lax Wendroff. Après un important travail d'implicitation, on obtient un code robuste permettant des calculs précis dans une large zone de nombre de Mach. Le schéma distributif ainsi obtenu, le schéma Lax Wendroff - PSI, permet de réduire l'entropie numérique et donc d'obtenir des calculs de traînées précis. Enfin, des résultats numériques obtenus à l'aide de ce schéma sont présentés et comparés avec un code Euler de référence, utilisant une approche de type Lax Wendroff.
\end{abstract}

AMS Subject Classification. 76M10.

Received: September 15, 1998. Revised: March 30, 1999 and May 25, 1999.

Keywords and phrases. 3D Euler equations, robust fluctuation splitting scheme, large range of Mach number, accurate flow computations.

* Part of this paper has been done in the framework of specific aeronautics research of the BRITE/EURAM Programme IDEMAS of the European Commission.

1 École Nationale des Ponts et Chaussées, 6 et 8 avenue Blaise Pascal, Cité Descartes, Champs sur Marne, 77455 Marne la Vallée Cedex 2, France.

${ }^{2}$ Dassault Aviation, DGT, DPR, DESA, 78 quai Marcel Dassault, 92214 Saint-Cloud, France.

e-mail: gilbert.roge@dassault-aviation.fr

(C) EDP Sciences, SMAI 1999 


\section{INTRODUCTION}

The computation of the inviscid flow over an aircraft using unstructured meshes is a routine task at Dassault Aviation.

Aerodynamics loads, flutter analysis, verification that the shock location does not impair the stability of the plane or the performance of the engines are among the applications of such a software.

The accurate shock location is obtained by coupling the inviscid solver with a boundary layer code to take into account the viscous effects.

For all these applications, numerical efficiency and accuracy are a must and the quality of the computation is judged by the aerodynamic forces, the precision of the shocks location and the pressure distribution on the airframe.

The methodology needs to be applicable to a wide Mach number range: from low subsonic to nearly hypersonic flows. The numerical ingredients must not depend upon the computational case and the reliability as well as the CPU efficiency are important in an industrial context.

In the 80's a lot of research and development took place and led to accurate and robust formulations, but there is still some room for improvement. For example, the accurate prediction of the drag coefficient may not be obtained due to some spurious entropy production.

Dassault Aviation has always been trying to improve the numerical methods used in its industrial codes. In 1995 [9], the development of the fluctuation splitting schemes showed an impressive potential, essentially in diminishing the spurious entropy.

In this paper, after a general presentation of the fluctuation splitting schemes, we present the fluctuation splitting scheme we have implemented in the industrial Euler code of Dassault Aviation and the numerical results we have obtained with this scheme.

\section{The FluCtuation SPlitting SCHEMES}

\subsection{The fluctuation splitting formalism}

In 1982 Roe introduced these new kind of numerical schemes and since then they have been developed by many others. The fluctuation splitting schemes, also called residual distribution schemes, have been largely presented in the literature, for instance in [5] or in [9].

The fluctuation splitting methodology for solving the scalar conservation law problem on an arbitrary triangulation of a domain $\Omega$ is recalled.

Let us consider the problem

$$
\left\{\begin{array}{l}
\frac{\partial \xi}{\partial t}+\nabla \cdot \mathcal{F}=0 \quad \forall(x, y, z) \in \Omega, \forall t>0 \\
\text { boundary conditions } \\
\text { initial conditions. }
\end{array}\right.
$$

Just like in linear finite element methods, the solution is approximated by a continuous function, varying linearly over each tetrahedron,

$$
\xi(x, y, z, t)=\sum_{i} \xi_{i}(t) \omega_{i}(x, y, z)
$$

where $\xi_{i}(t)$ is the value of $\xi$ at node $N_{i}$, and $\omega_{i}$ the linear shape function equal to unity at $\left(x_{i}, y_{i}, z_{i}\right)$ and equal to zero outside the support of all tetrahedra meeting at node $N_{i}$. 
The integration over an element $\mathrm{T}$ leads to

$$
\iiint_{T} \frac{\partial \xi}{\partial t} \mathrm{~d} V=-\iiint_{T} \vec{\lambda} \cdot \nabla \xi \mathrm{d} V
$$

with

$$
\vec{\lambda}=\frac{\partial \mathcal{F}}{\partial \xi}
$$

Using a first order in time approximation, we can write the left-hand side of (3) in the local element numbering as

$$
\iiint_{T} \frac{\partial \xi}{\partial t} \mathrm{~d} V=\frac{V_{T}}{4}\left[\frac{\xi_{1}^{n+1}-\xi_{1}^{n}}{\Delta t}+\frac{\xi_{2}^{n+1}-\xi_{2}^{n}}{\Delta t}+\frac{\xi_{3}^{n+1}-\xi_{3}^{n}}{\Delta t}+\frac{\xi_{4}^{n+1}-\xi_{4}^{n}}{\Delta t}\right]
$$

where $V_{T}$ denotes the volume of the element $T$, and $\Delta t$ is a time-step parameter.

For the right-hand side, we choose an approximation in order to compute the residual $\Phi^{T}=\iiint_{T} \vec{\lambda} \cdot \nabla \xi \mathrm{d} V$. The idea of the fluctuation splitting schemes is then to split the residual in

$$
\Phi^{T}=\Phi_{1}^{T}+\Phi_{2}^{T}+\Phi_{3}^{T}+\Phi_{4}^{T}
$$

The fluctuation splitting method consists in distributing fractions of the residual. This leads to the explicit scheme

$$
\sum_{T \mid N_{i} \in T} \frac{V_{T}}{4} \frac{\xi_{i}^{n+1}-\xi_{i}^{n}}{\Delta t}=-\sum_{T \mid N_{i} \in T} \Phi_{i}^{T}
$$

or, in an equivalent way,

$$
\xi_{i}^{n+1}=\xi_{i}^{n}-\frac{\Delta t}{V_{i}} \sum_{T \mid N_{i} \in T} \Phi_{i}^{T}
$$

after having introduced $V_{i}=\sum_{T \mid N_{i} \in T} V_{T} / 4$ which is no more than the volume of the dual cell (Fig. 1) at node $N_{i}$.

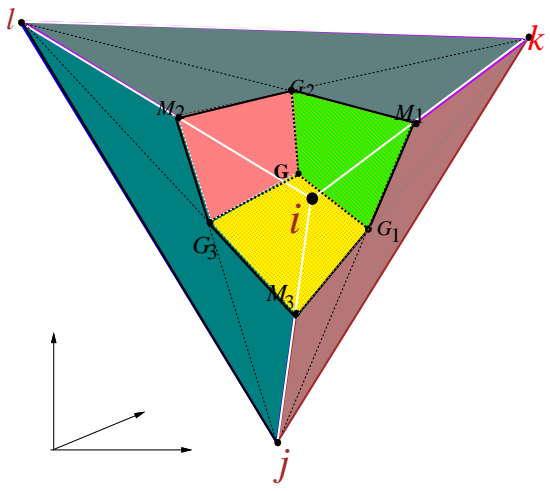

Figure 1. Footprint of the dual cell onto a typical tetrahedron containing node $N_{i}$. 
It is often useful to introduce the coefficients $\beta_{i}^{T}$ defined by $\Phi_{i}^{T}=\beta_{i}^{T} \Phi^{T}$. We require that $\sum_{i \mid N_{i} \in T} \beta_{i}^{T}=1$ for consistency.

Fluctuation splitting schemes were first developed for the scalar advection equation. Then, the problem of the extension of the scalar schemes to systems had to be solved.

This problem is easily solved when the system is diagonalisable. In this case, scalar schemes can be used for each scalar equations of the diagonalised system. But, here, our aim is to solve the Euler equations, which are not diagonalisable.

For such non-diagonalisable systems, three main orientations are identified. One consists in a formal extension of the scalar scheme. An application of this idea is found for the N-scheme (Narrow scheme) in [3]. Another orientation, first introduced by Roe in 1986 [12], is to decompose the initial residual $\Phi_{T}$ as a sum of simple wave solutions. The last one is a characteristic based splitting such as initially proposed by Deconinck et al. [4], or such as the hyperbolic/elliptic splitting described in this paper.

\subsection{The properties of the distributions}

We recall here the main properties of the fluctuation splitting schemes and the conditions they impose on the distributions $\Phi_{i}^{T}$.

Before listing these properties, we need to introduce, for each node $N_{i}$ of an element $T, k_{i}=(1 / 3) \vec{\lambda} \cdot \vec{\nu}_{i}$. $\vec{\nu}_{i}$ denotes the vector normal to the face opposite to node $N_{i}$ in tetrahedron $T$, pointing toward node $N_{i}$, and scaled by the surface of the face (Fig. 2).

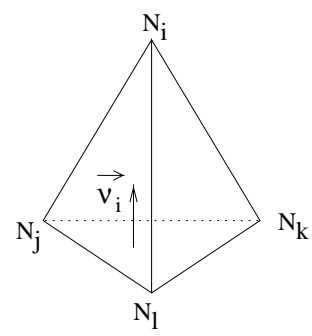

FiguRE 2. Tetrahedron with the inward vector normal $\vec{\nu}_{i}$.

Thus, one has $\sum_{i=1}^{4} \vec{\nu}_{i}=\overrightarrow{0}$ and $\sum_{i=1}^{4} k_{i}=0$.

First, a fluctuation splitting scheme is said to be upwind when the distributions $\Phi_{i}^{T}$ satisfy

$$
\text { (U) } \beta_{i}^{T}=0 \text { if } k_{i}<0 .
$$

This property is called upwinding because it is the exact extension of the upwinding notion for the resolution of the one dimensional convection equation.

Another property is positivity. A fluctuation splitting scheme is said to be positive if the value at a new time-step can be written as a convex sum of values at the previous time-step,

$$
(\mathcal{P}) \quad \xi_{i}^{n+1}=\sum_{k} c_{k} \xi_{k}^{n} \text { with } c_{k} \geq 0 \quad \forall k
$$

together with the consistency condition $\sum_{k} c_{k}=1$. 
The last important property is to be linearity preserving. This property is strongly tied to second order accuracy. A fluctuation splitting scheme is said to be linearity preserving when

$$
(\mathcal{L P}) \quad \Phi_{i}^{T} \rightarrow 0 \text { when } \Phi^{T} \rightarrow 0
$$

a sufficient condition to guaranty this property being that $\beta_{i}^{T}$ is bounded.

\section{A CRuCial Decomposition of the Euler EQUations}

In this section, we recall the hyperbolic/elliptic decomposition of the Euler equations. This decomposition, inspired by the Deconinck-Hirsch-Peuteman decomposition [4], has been exposed in [9] in the two dimensional case and in [3] in the three dimensional case. This decomposition is crucial for the scheme presented in the next section.

The Euler equations are usually written with a conservative form using the variable

$$
W=\left(\begin{array}{c}
\rho \\
\rho u \\
\rho v \\
\rho w \\
\rho e
\end{array}\right)
$$

where $\rho$ represents the gas density, $u, v$ and $w$ are the $x-, y-$ and $z$-components of the velocity vector $\vec{u}$ and $e$ is the specific total energy.

This conservative form is

$$
\frac{\partial F}{\partial x}+\frac{\partial G}{\partial y}+\frac{\partial H}{\partial z}=0
$$

with

$$
F=\left(\begin{array}{c}
\rho u \\
\rho u^{2}+p \\
\rho u v \\
\rho u w \\
\rho u h
\end{array}\right), \quad G=\left(\begin{array}{c}
\rho v \\
\rho u v \\
\rho v^{2}+p \\
\rho v w \\
\rho v h
\end{array}\right), \quad H=\left(\begin{array}{c}
\rho w \\
\rho u w \\
\rho v w \\
\rho w^{2}+p \\
\rho w h
\end{array}\right),
$$

$p$ the pressure, and $h$ the specific total enthalpy.

We denote

$$
\mathcal{F}=F \overrightarrow{1}_{x}+G \overrightarrow{1}_{y}+H \overrightarrow{1}_{z}
$$

In the fluctuation splitting context, it is useful to consider the quasi linear form of the Euler equations

$$
A \frac{\partial W}{\partial x}+B \frac{\partial W}{\partial y}+C \frac{\partial W}{\partial z}=0
$$

with

$$
A=\frac{\partial F}{\partial W}, B=\frac{\partial G}{\partial W}, C=\frac{\partial H}{\partial W}
$$

and we write

$$
\mathcal{A}=A \overrightarrow{1}_{x}+B \overrightarrow{1}_{y}+C \overrightarrow{1}_{z} .
$$


Although we want to solve the steady Euler equations, we consider the unsteady Euler equations because we use a pseudo unsteady process with local time-stepping to reach steady states. Thus, we consider

$$
\frac{\partial W}{\partial t}+A \frac{\partial W}{\partial x}+B \frac{\partial W}{\partial y}+C \frac{\partial W}{\partial z}=0
$$

Two changes of variables have to be made in order to obtain the hyperbolic/elliptic decomposition.

In a first step, we use the symmetrizing variables:

$$
\partial Q=\left(\begin{array}{c}
\frac{\partial p}{\rho a} \\
\partial u \\
\partial v \\
\partial w \\
\partial p-a^{2} \partial \rho
\end{array}\right)=\varpi \partial U \text { with } U=\left(\begin{array}{c}
\rho \\
u \\
v \\
w \\
p
\end{array}\right) \text { and } \varpi=\left[\begin{array}{ccccc}
0 & 0 & 0 & 0 & 1 / \rho a \\
0 & 1 & 0 & 0 & 0 \\
0 & 0 & 1 & 0 & 0 \\
0 & 0 & 0 & 1 & 0 \\
-a^{2} & 0 & 0 & 0 & 1
\end{array}\right]
$$

where $a$ is the speed of sound.

With the variable $Q$, the Euler equations become

$$
\frac{\partial Q}{\partial t}+\tilde{A} \frac{\partial Q}{\partial x}+\tilde{B} \frac{\partial Q}{\partial y}+\tilde{C} \frac{\partial Q}{\partial z}=0
$$

with

$$
\tilde{A}=\left[\begin{array}{ccccc}
u & a & 0 & 0 & 0 \\
a & u & 0 & 0 & 0 \\
0 & 0 & u & 0 & 0 \\
0 & 0 & 0 & u & 0 \\
0 & 0 & 0 & 0 & u
\end{array}\right], \tilde{B}=\left[\begin{array}{ccccc}
v & 0 & a & 0 & 0 \\
0 & v & 0 & 0 & 0 \\
a & 0 & v & 0 & 0 \\
0 & 0 & 0 & v & 0 \\
0 & 0 & 0 & 0 & v
\end{array}\right] \text { and } \tilde{C}=\left[\begin{array}{ccccc}
w & 0 & 0 & a & 0 \\
0 & w & 0 & 0 & 0 \\
0 & 0 & w & 0 & 0 \\
a & 0 & 0 & w & 0 \\
0 & 0 & 0 & 0 & w
\end{array}\right]
$$

After this symmetrization of the Euler equations, we introduce the second change of variables

$$
\partial \tilde{W}=\left(\begin{array}{c}
\frac{\beta}{\rho a} \partial p+M \vec{s} \cdot \partial \vec{u} \\
\frac{\beta}{\rho a} \partial p-M \vec{s} \cdot \partial \vec{u} \\
M \vec{t} \cdot \partial \vec{u} \\
\frac{\partial p}{\rho a}+M \vec{n} \cdot \partial \vec{u} \\
\partial p-a^{2} \partial \rho
\end{array}\right)=L \partial Q \text { with } L=\left[\begin{array}{ccccc}
\beta & M s_{x} & M s_{y} & M s_{z} & 0 \\
\beta & -M s_{x} & -M s_{y} & -M s_{z} & 0 \\
0 & M t_{x} & M t_{y} & M t_{z} & 0 \\
1 & M n_{x} & M n_{y} & M n_{z} & 0 \\
0 & 0 & 0 & 0 & 1
\end{array}\right]
$$

where $M$ is the free stream Mach number, $\beta$ defined as

$$
\beta=\sqrt{\max \left(\iota^{2},\left|M^{2}-1\right|\right)}
$$

with $\iota=0.05$, like in [9], $\vec{n}=\frac{\vec{u}}{\|\vec{u}\|}$ and $(\vec{n}, \vec{s}, \vec{t})$ is an orthonormal direct base.

This change of variables leads to

$$
\frac{\partial W}{\partial t}+\frac{\partial W}{\partial Q}\left[\tilde{A} R \frac{\partial \tilde{W}}{\partial x}+\tilde{B} R \frac{\partial \tilde{W}}{\partial y}+\tilde{C} R \frac{\partial \tilde{W}}{\partial z}\right]=0
$$


with $R=L^{-1}$.

In order to obtain the hyperbolic/elliptic decomposition, we have to use the preconditionning matrix which Van Leer et al. introduced in 1991 [22] and extended to the 3D case by Bonfiglioli et al. in 1996 [3]

$$
P=\frac{1}{q}\left[\begin{array}{ccccc}
\frac{\chi M^{2}}{\beta^{2}} & \frac{-\chi M u}{\beta^{2} q} & \frac{-\chi M v}{\beta^{2} q} & \frac{-\chi M w}{\beta^{2} q} & 0 \\
\frac{-\chi M u}{\beta^{2} q} & \eta \frac{u^{2}}{q^{2}}+\chi & \eta \frac{u v}{q^{2}} & \eta \frac{u w}{q^{2}} & 0 \\
\frac{-\chi M v}{\beta^{2} q} & \eta \frac{u v}{q^{2}} & \eta \frac{v^{2}}{q^{2}}+\chi & \eta \frac{v w}{q^{2}} & 0 \\
\frac{-\chi M w}{\beta^{2} q} & \eta \frac{u w}{q^{2}} & \eta \frac{v w}{q^{2}} & \eta \frac{w^{2}}{q^{2}}+\chi & 0 \\
0 & 0 & 0 & 0 & 1
\end{array}\right]
$$

with $q=\|\vec{u}\|$ and $\chi, \eta$ defined as

$$
\chi=\frac{\beta}{\max (M, 1)}, \eta=\frac{\chi+\beta^{2}}{\beta^{2}}-\chi .
$$

With this matrix, we write $(25)$ in an equivalent form

$$
\frac{\partial W}{\partial t}+\frac{\partial W}{\partial Q} P^{-1} R\left[L P \tilde{A} R \frac{\partial \tilde{W}}{\partial x}+L P \tilde{B} R \frac{\partial \tilde{W}}{\partial y}+L P \tilde{C} R \frac{\partial \tilde{W}}{\partial z}\right]=0 .
$$

This new form leads to

$$
\frac{\partial W}{\partial t}+\frac{\partial W}{\partial Q} P^{-1} R \mathcal{R}(\tilde{W})=0
$$

with

$$
\mathcal{R}(\tilde{W})=\left[\begin{array}{ccccc}
\chi \mu^{+} \vec{n}+\frac{\chi}{\beta} \vec{s} & \chi \mu^{-} \vec{n} & \frac{\chi}{\beta} \vec{t} & 0 & 0 \\
\chi \mu^{-} \vec{n} & \chi \mu^{+} \vec{n}-\frac{\chi}{\beta} \vec{s} & \frac{\chi}{\beta} \vec{t} & 0 & 0 \\
\frac{\chi}{2 \beta} \vec{t} & \frac{\chi}{2 \beta} \vec{t} & \chi \vec{n} & 0 & 0 \\
0 & 0 & 0 & \vec{n} & 0 \\
0 & 0 & 0 & 0 & \vec{n}
\end{array}\right] \nabla \tilde{W} \text { and } \mu^{ \pm}=\frac{M^{2}-1 \pm \beta^{2}}{2 \beta^{2}}
$$

Thus, we have obtained an equivalent form of the Euler equations (29), at least locally over each element $T$.

In this new form, two different parts can be distinguished. The first three equations of (30) constitute a subsystem which is independent of the last two equations and is called the acoustic subsystem. The last two ones are entirely decoupled scalar advection equations.

Using this decomposition, we can treat the two parts in different ways. This is the object of the next section, where we introduce the Lax Wendroff - PSI scheme.

\section{The Lax Wendroff - PSI scheme}

We present here the Lax Wendroff - PSI scheme. This scheme is based upon the matrix-scalar decomposition of the residual. Indeed, the divergence of the fluxes can be written as the sum of the residual of the acoustic 
subsystem and of the residuals of the last two scalar advection equations:

$$
\frac{\partial F}{\partial x}+\frac{\partial G}{\partial y}+\frac{\partial H}{\partial z}=\underbrace{\left(r^{1}, r^{2}, r^{3}\right)\left[\tilde{a} \nabla\left(\begin{array}{c}
\tilde{W}^{1} \\
\tilde{W}^{2} \\
\tilde{W}^{3}
\end{array}\right)\right]}_{\Phi_{\text {coupled }}}+\underbrace{\vec{\lambda}^{4} \cdot \tilde{W}^{4} r^{4}}_{\Phi_{\text {enthal }}}+\underbrace{\vec{\lambda}^{5} \cdot \tilde{W}^{5} r^{5}}_{\Phi_{\text {entrop }}}
$$

where $r^{i}$ are the vectors of the matrix $\frac{\partial W}{\partial Q} P^{-1} R, \vec{\lambda}^{l}$ the advection vectors of the last two scalar equations (in practice, $\vec{\lambda}^{4}=\vec{\lambda}^{5}=\vec{n}$ ) and

$$
\tilde{a}=\left[\begin{array}{ccc}
\chi \mu^{+} \vec{n}+\frac{\chi}{\beta} \vec{s} & \chi \mu^{-} \vec{n} & \frac{\chi}{\beta} \vec{t} \\
\chi \mu^{-} \vec{n} & \chi \mu^{+} \vec{n}-\frac{\chi}{\beta} \vec{s} & \frac{\chi}{\beta} \vec{t} \\
\frac{\chi}{2 \beta} \vec{t} & \frac{\chi}{2 \beta} \vec{t} & \chi \vec{n}
\end{array}\right]=\left(\tilde{a}_{x}, \tilde{a}_{y}, \tilde{a}_{z}\right) .
$$

The strategy of the Lax Wendroff - PSI scheme (LW-PSI scheme) is to split the acoustic residual, $\Phi_{\text {coupled }}$, with a matrix fluctuation splitting and the two scalar residuals, $\Phi_{\text {enthal }}$ and $\Phi_{\text {entrop }}$ with a scalar fluctuation splitting scheme.

In the LW-PSI scheme, the matrix Lax Wendroff scheme is used to split $\Phi_{\text {coupled }}$ and the PSI scheme is used to split $\Phi_{\text {enthal }}$ and $\Phi_{\text {entrop }}$.

\subsection{The matrix Lax Wendroff scheme}

The Lax Wendroff scheme can be formulated as a fluctuation splitting scheme, as shown by Roe in 1987 [13]. Thus, in the scalar case, the Lax Wendroff scheme corresponds to the distribution

$$
\beta_{i}^{L W, T}=\frac{1}{4}+\frac{\Delta t}{2 V_{T}} k_{i}
$$

where $\Delta t$ is a time-step parameter.

The matrix Lax Wendroff scheme is obtained with a formal generalisation of (33). Thus, we have

$$
\beta_{i}^{L W, T}=\frac{1}{4} \operatorname{Id}+\frac{\Delta t}{2 V_{T}} K_{i}
$$

where $K_{i}$ is a matrix generalisation of the scalar $k_{i}$

$$
K_{i}=\frac{1}{3}\left[\tilde{a}_{x} \nu_{i_{x}}+\tilde{a}_{y} \nu_{i_{y}}+\tilde{a}_{z} \nu_{i_{z}}\right] .
$$

The Rudgyard extension of the time-step $\Delta t[10,14]$ allows to obtain a matrix Lax Wendroff scheme which does not depend on $\Delta t$ :

$$
\beta_{i}^{L W, T}=\frac{1}{4} \operatorname{Id}+\frac{\mu_{\text {cell }}}{2} K_{i}\left(\sum_{j}\left|K_{j}\right|\right)^{-1}
$$

where $\mu_{\text {cell }}$ is a constant taken to 1 and $\left|K_{i}\right|$ is the matrix which has the same eigenvectors as $K_{i}$ but whose eigenvalues are the absolute value of those of $K_{i}$. 


\subsection{The PSI scheme}

The PSI (Positive Streamwise Invariant) scheme was introduced by Struijs et al. in 1991 [21]. In 1995, Sidilkover and Roe [18] showed that this PSI scheme can be obtained by applying the Min Mod limiter function to the N-scheme. The Min Mod limiter function is exposed in [17], and the N-scheme is a very popular fluctuation splitting scheme to which Sildikover gave its name in 1989 [19]. The later scheme has been well extended to the three dimensional case by Bonfiglioli et al. in 1996 [3].

This scheme is a scalar upwinding fluctuation splitting scheme which is positive and linearity preserving. These properties make this scheme one of the most performant one to solve the scalar advection problem. The handicap of this scheme is that it can not be extended to the system case in a satisfactory way.

This scheme could be replaced, in two dimensions, by the N-SUPG scheme of Abgrall (private communication). For all the 2D numerical simulations performed, the PSI and the N-SUPG gave the same results.

\subsection{An additional viscous term}

The first uses of the LW-PSI scheme showed an oscillatory behavior in two dimensions. In three dimensions, this behavior became a main obstacle for computations. For instance, a pure subsonic computational case over a wing could not be realized with this scheme as soon as Mach number was higher than 0.5.

As a matter of fact, this behavior is not surprising since we use a Lax Wendroff scheme to split the acoustic residual, and the Lax Wendroff scheme is not monotone and generates oscillations near the shocks.

In order to reduce this oscillatory behavior, we add a viscous term to the LW-PSI scheme. This term is based on the second order term which can be found in the Peraire's scheme $[8,11,16]$. This viscous term is nearly a laplacian, which is decomposed over each element. Then, we evaluate the necessary viscosity in order to dump the fastest wave.

In order to limit the degradation of the LW-PSI scheme, we use the pressure sensor designed by Jameson and Schmidt in 1985 [8]. This pressure sensor is calculated, for each edge, as

$$
p s_{i, j}=\min \left(p s_{i}, p s_{j}\right) \text { with } p s_{i}=\frac{\left|p_{j}-p_{i}-\nabla p_{i} \cdot \overrightarrow{N_{i} N_{j}}\right|}{\left|p_{j}+p_{i}-\nabla p_{i} \cdot \overrightarrow{N_{i} N_{j}}\right|}
$$

where $p_{i}$ is the pressure at the node $N_{i}$.

This pressure sensor helps us to add viscosity only near the shocks. In practice, the viscous term we added is

$$
\Phi_{\mathrm{visc}}^{i}=\left(r^{1}, r^{2}, r^{3}\right) \frac{1}{2 \sum_{T \mid N_{i} \in T} V_{T}}\left(\sum_{T} \sum_{\mid N_{i} \in T} \lambda_{j, j} \epsilon_{i, j} V_{T}\left(\nabla \tilde{W} \cdot \overrightarrow{N_{i} N_{j}}\right)\right)
$$

with

$$
\begin{gathered}
\lambda_{i}=\max \left[\left|\vec{\lambda}^{1} \cdot \vec{\nu}_{i}\right|,\left|\vec{\lambda}^{2} \cdot \vec{\nu}_{i}\right|,\left|\vec{\lambda}^{3} \cdot \vec{\nu}_{i}\right|\right], \quad \lambda_{i, j}=\frac{\lambda_{i}+\lambda_{j}}{2} \\
\vec{\lambda}^{1}=\chi \vec{n}+\frac{\chi}{\beta} \vec{s}, \quad \vec{\lambda}^{2}=\chi \vec{n}-\frac{\chi}{\beta} \vec{s}, \quad \vec{\lambda}^{3}=\frac{\chi}{\beta} \vec{t}
\end{gathered}
$$

and

$$
\epsilon_{i, j}=\min \left(1, \kappa p s_{i, j}\right) \text { with } \kappa=15 .
$$




\subsection{The Roe linearization}

Until now, it has been proceeded as if the Euler equations were linear. The extension of the fluctuation splitting method to non linear conservation laws is largely discussed in [9] and the conclusion is that the linearization $\mathcal{L}(\bar{Z})[6,20]$ has to be used as far as we have to get a constant approximation of whatever quantity.

We just recall here that this linearization is a generalisation of the Roe average. This linearization is based on the assumption that the parameter vector $Z=\sqrt{\rho}(1, u, v, w, h)$ varies linearly over each element. This choice of $Z$ is such that $W$ and the fluxes $F, G, H$ are quadratic in the components of $Z$ and so the jacobians of these four quantities are linear in the components of $Z$.

Thus, we denote $\breve{u}, \breve{a}, \ldots$, the variables which come from the Roe linearization.

Before going on, we have to explain how we take into account the boundary conditions.

\subsection{The boundary conditions}

We choose a treatment of the boundary conditions which allows us to use all the subroutines already written for our finite volume schemes, for instance those of our reference code. This treatment is a general treatment which can be used for every fluctuation splitting scheme.

The finite volume schemes lead to the decomposition

$$
\begin{aligned}
\iiint_{\Omega} \frac{\partial W}{\partial t} \varphi & =-\iiint_{\Omega} \nabla \cdot \mathcal{F} \varphi \\
& =\iiint_{\Omega} \mathcal{F} \cdot \nabla \varphi-\iint_{\partial \Omega} \mathcal{F} \cdot \vec{\nu} \varphi
\end{aligned}
$$

where $\varphi$ is a test function. Then a weak formulation of boundary conditions is used to compute $\int_{\partial \Omega} \mathcal{F} \cdot \vec{\nu} \varphi$.

In the fluctuation splitting formalism, $\iiint_{\Omega} \nabla \cdot \mathcal{F} \varphi$ is directly computed. In order to do the same treatment as in the finite volume case, we write

$$
\begin{aligned}
\iiint_{\Omega} \frac{\partial W}{\partial t} \varphi & =-\iiint_{\Omega} \mathcal{A} \nabla W \varphi \\
& =-\iiint_{\Omega} \mathcal{A} \nabla W \varphi+\iint_{\partial \Omega} \mathcal{F} \cdot \vec{\nu} \varphi-\iint_{\partial \Omega} \mathcal{F} \cdot \vec{\nu} \varphi .
\end{aligned}
$$

Thus, we compute in a first time

$$
\underbrace{-\iiint_{\Omega} \mathcal{A} \nabla W \varphi}_{\text {fluctuation splitting }}+\underbrace{\iint_{\partial \Omega} \mathcal{F} \cdot \vec{\nu} \varphi}_{\text {centered treatment }}
$$

and then we add $-\iint_{\partial \Omega} \mathcal{F} \cdot \vec{\nu} \varphi$, which is computed with a weak formulation of the boundary conditions.

\section{The TIME-INTEGRATion TO THE STEADY STATE}

The explicit scheme used to compute Euler steady states has been presented. But our industrial constraints are such that explicit schemes are not fast enough. This is the reason why implicit schemes have to be used.

The way, chosen to obtain an implicit scheme, is discussed here.

The explicit scheme can be written as

$$
W_{i}^{n+1}=W_{i}^{n}-\frac{\Delta t_{i}}{V_{i}} \operatorname{Res}_{i}\left(W^{n}\right)
$$

where $\operatorname{Res}_{i}\left(W^{n}\right)$ is the residual which is affected to node $N_{i}$ at the iteration $n$. 
Any implicit scheme can be written as

$$
W_{i}^{n+1}=W_{i}^{n}-\frac{\Delta t_{i}}{V_{i}} \operatorname{Res}_{i}\left(W^{n+1}\right) .
$$

As far as we are concerned, a linearized implicit scheme is used. Thus, we write

$$
\operatorname{Res}_{i}\left(W^{n+1}\right) \simeq \operatorname{Res}_{i}\left(W^{n}\right)+\frac{\partial \operatorname{Res}_{i}}{\partial W}\left(W^{n}\right)\left(W^{n+1}-W^{n}\right)
$$

where $\partial \operatorname{Res}_{i} / \partial W$ is the jacobian matrix of $\operatorname{Res}_{i}$

Finally, we use

$$
W^{n+1}=W^{n}+\left(\operatorname{Id}+\frac{\Delta t}{V} \frac{\partial \operatorname{Res}}{\partial W}\left(W^{n}\right)\right)^{-1}\left(-\frac{\Delta t}{V} \operatorname{Res}\left(W^{n}\right)\right)
$$

where $\Delta t / V$ is a block diagonal matrix, whose each block is $\left(\Delta t_{i} / V_{i}\right) \mathrm{Id}$.

Once more, we have to face our industrial constraints. As a matter of fact, we can not store the matrix Id $+\frac{\Delta t}{V} \frac{\partial R e s}{\partial W}\left(W^{n}\right)$ and inverse it for each iteration. Because we have to guaranty a low storage, and efficiency, we have to solve

$$
\left(\operatorname{Id}+\frac{\Delta t}{V} \frac{\partial \operatorname{Res}}{\partial W}\left(W^{n}\right)\right) y=-\frac{\Delta t}{V} \operatorname{Res}\left(W^{n}\right)
$$

at each iteration of the unsteady process.

The resolution of this linear system is the issue of the next section.

\subsection{The resolution of the linear system of the implicit scheme}

In order to solve this linear system, we use a GMRES iterative solver [15].

Until the end of this section, we denote $A=\operatorname{Id}+\frac{\Delta t}{V} \frac{\partial \operatorname{Res}}{\partial W}\left(W^{n}\right)$ and thus we denote the linear system $A x=b$.

The use of GMRES solver requires computations of $A x$ and a preconditioner.

The difficult point in the computations of $A x$ are those of $\frac{\partial \mathrm{Res}}{\partial W}\left(W^{n}\right) x$. In order to realize these computations we use a software designed by INRIA: Odyssee [7]. This software is able to perform automatic differentiation of FORTRAN subroutines: it generates the subroutine for the computation of $\frac{\partial \mathrm{Res}}{\partial W}\left(W^{n}\right) x$ from the subroutine of $\operatorname{Res}\left(W^{n}\right)$. With this software, we have been able to compute $\frac{\partial \operatorname{Res}}{\partial W}\left(W^{n}\right) x$ in a very precise way.

Concerning the other point, we use a left block diagonal preconditionner and the computation of this preconditionning matrix is also realised with Odyssee.

The last point we have to describe is the way we compute the time-step.

\subsection{The time-stepping}

Because our objective is to reach steady state, we can use a local time-step. We discuss here the choice of $\Delta t_{i}$ we made.

First of all, the PSI-scheme is positive under the CFL condition [9]

$$
\Delta t_{i} \leq \min _{l}\left(\frac{V_{i}}{\sum_{T \mid N_{i} \in T} \max \left(0,\left|\vec{\lambda}^{l} \cdot \vec{\nu}_{i}\right|\right)}\right)
$$


with

$$
\overrightarrow{\check{\lambda}}^{l}=\check{u} \vec{\lambda}^{l}
$$

We recall that $\check{u}$ comes from the Roe linearization (Sect. 4.4).

This gives a first constraint for the choice of the time-step. But, for reasons of robustness, we choose a more restrictive time-step

$$
\Delta t_{i}=f(\epsilon) \frac{V_{i}}{\sum_{T \mid N_{i} \in T}(\check{u}+\check{a})\left\|\vec{\nu}_{i}\right\| \max \left(1, \chi\left(1+\frac{1}{\beta}\right)\right)}
$$

where

$$
\epsilon=\frac{1}{3 V_{i}} \sum_{T \mid N_{i} \in T} V_{T} \frac{\epsilon_{i, j}+\epsilon_{i, k}+\epsilon_{i, l}}{3}
$$

and

$$
f(\epsilon)=\frac{\sqrt{4+\epsilon^{2}}-\epsilon}{2}
$$

The function $f$ was derived from the stability analysis of the one dimensional Lax Wendroff scheme including the additional viscosity. This scheme can be written as

$$
\underbrace{\frac{\xi_{i}^{n+1}-\xi_{i}^{n}}{\Delta t}+\tilde{a} \frac{\xi_{i+1}^{n}-\xi_{i-1}^{n}}{2 \Delta x}-\tilde{a}^{2} \frac{\Delta t}{2 \Delta x^{2}} \mathcal{D}\left(\xi^{n}\right)}_{\text {Lax Wendroff }}-\underbrace{\epsilon \frac{\tilde{a}}{2 \Delta x} \mathcal{D}\left(\xi^{n}\right)}_{\text {viscosity }}=0
$$

with

$$
\mathcal{D}\left(\xi^{n}\right)=\xi_{i+1}^{n}-2 \xi_{i}^{n}+\xi_{i-1}^{n}
$$

and where we have called in the two former equations $\epsilon$ the coefficient based upon the Jameson pressure sensor.

A classical method gives the following stability criterium

$$
\frac{\tilde{a} \Delta t}{\Delta x} \leq \frac{\sqrt{4+\epsilon^{2}}-\epsilon}{2}
$$

and so $f$.

Indeed, $\Delta t_{i}$ is the time-step we use with the explicit version of the code. In the implicit one, we multiply this time-step by a factor which is taken between 1 and 100 .

The work devoted to the implicitation of the Lax Wendroff - PSI scheme was very necessary in order to obtain a fast solver, and thus to consider industrial applications. Moreover, each of the components of this work are crucial in order to obtain an efficient and robust code. We have to precise that, when we used the implicit version, we had to take the time-step defined by (52-54) in order to get a solution. A convergence history obtained with this implicit time-stepping strategy is shown in Figure 9.

All the different aspects of our scheme have been presented. We now focus our attention on the results obtained with this scheme. 


\section{Numerical Results}

The capabilities of the code are illustrated by several numerical examples.

In order to be able to validate the results, we compare them to those obtained with the Lax Wendroff scheme of Dassault Aviation. From now on, we denote LW-PSI the Lax Wendroff - PSI scheme and LX the reference Lax Wendroff scheme.

The LX scheme is not a matrix Lax Wendroff scheme like the LW-PSI scheme. This LX scheme, first introduced by Billey in 1984 [2], is a predictor-corrector scheme which can be written in two steps

$$
\text { Step } 1 \text { (prediction): } \quad W_{p}^{n}(T)=\frac{1}{V_{T}}\left[\iiint_{T} W^{n} \mathrm{~d} V-\alpha \Delta t_{\text {pred }} \iint_{\partial T} \mathcal{F}\left(W^{n}\right) \cdot \vec{\nu} \mathrm{d} S\right]
$$

and

$$
\text { Step } 2 \text { (correction): } \Phi_{i}^{T}=\beta_{1} \iiint_{T}^{*} \mathcal{F}\left(W^{n}\right) \cdot \nabla \varphi_{i}+\beta_{2} \iiint_{T}^{* *} \mathcal{F}\left(W_{p}^{n}\right) \cdot \nabla \varphi_{i}-\text { A.V., }
$$

where $\alpha, \Delta t_{\text {pred }}, \beta_{1}, \beta_{2}$ are appropriate constants, $\iint^{*}$ and $\iint^{* *}$ represent two different approximations to compute the integrals, and the term A.V. is an additional viscosity term which looks like the term we have introduced in Section 4.3.

Before going on, we have to precise that the LX scheme is considered validated.

\subsection{Subsonic wing}

In this test case, we compute a subsonic flow around the ONERA M6 wing, at a free stream Mach number equal to $M_{\infty}=0.2$, and with no incidence.

The mesh (Fig. 3) has 27499 nodes and 152096 tetrahedra.

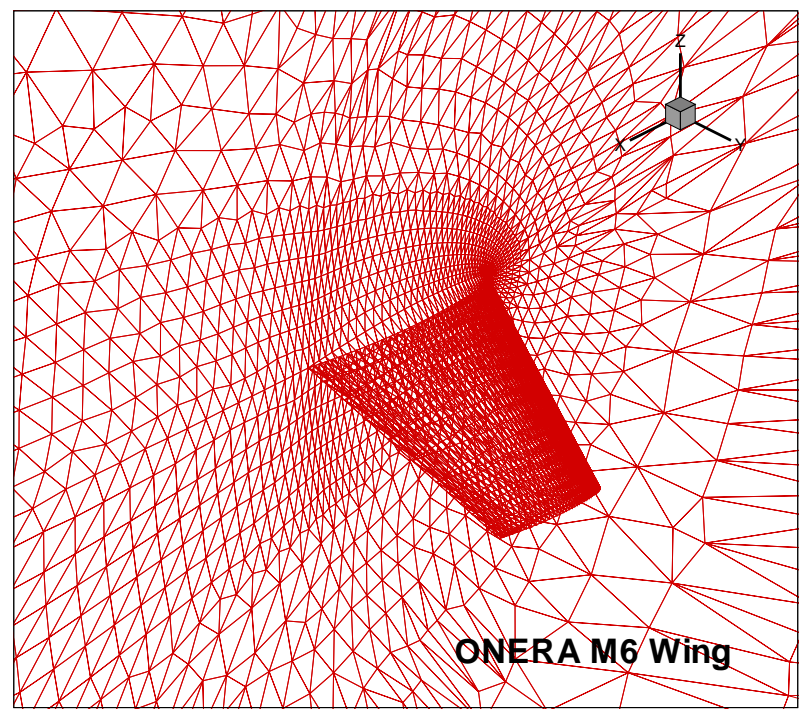

FIgURE 3. Partial view of the mesh of the ONERA M6 wing. 
First, we plot the entropy deviation $\Sigma$ along a profile, located at $60 \%$ on the wing span (Fig. 4).

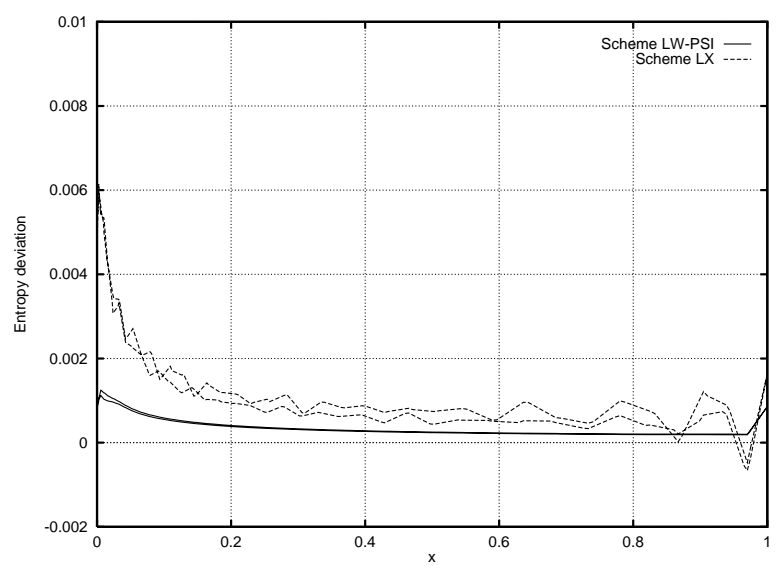

Figure 4. Comparison of $\Sigma$ along a profile.

$\Sigma$ is defined by

$$
\Sigma=\frac{p / p_{\infty}}{\left(\rho / \rho_{\infty}\right)^{\gamma}}-1 .
$$

We recall that $\Sigma$ should be equal to zero in subsonic Euler computations.

We can notice that the LW-PSI scheme generates much less numerical entropy than the LX scheme. This is illustrated in Figure 5 where absolute value of $\Sigma$ is plotted over the wing.

\section{Plot of absolute deviation entropy}

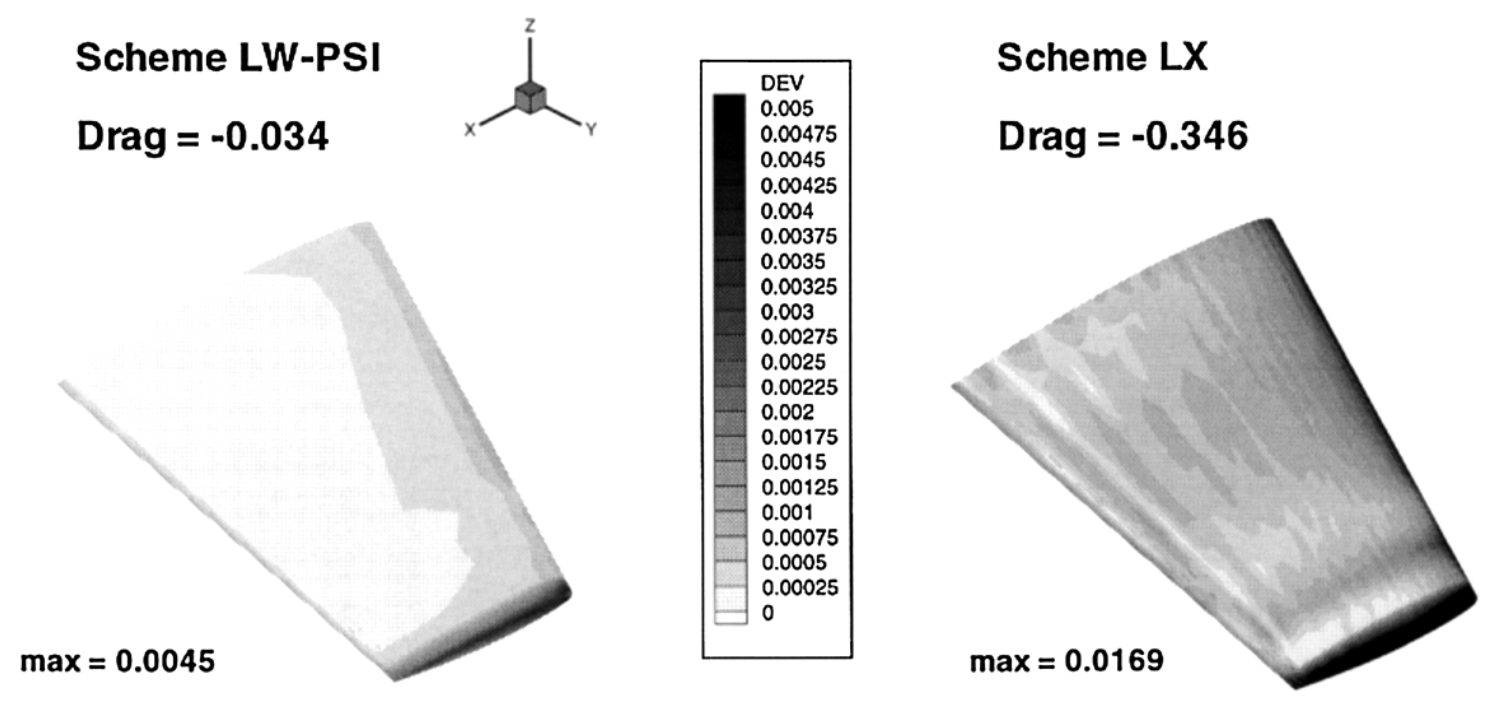

Figure 5. Comparison of absolute entropy deviation.

We point out the difference of numerical entropy at the stagnation line (Fig. 4). 
These more accurate entropy deviation allows to compute drag coefficients more accurately. Indeed, in this test case, the drag is theoretically null and the computed drag is ten times less important with the LW-PSI scheme than with the LX scheme.

\subsection{Transonic wing}

We now investigate the performances of the LW-PSI scheme for transonic flows.

We use the same mesh as in Section 6.1, and we take $M_{\infty}=0.88$ and $6^{\circ}$ for the incidence.

We choose to plot the pressure coefficient $C_{p}$ and the entropy deviation $\Sigma$ along the wing at the profile located at $60 \%$ of the span from the symmetry plane.

The $C_{p}$ distributions (Fig. 6) show that the LW-PSI scheme allows a more precise location of the shock than the LX scheme. We can also notice that oscillations have been reduced near the shock, thanks to the additional viscous term we have introduced at Section 4.3.

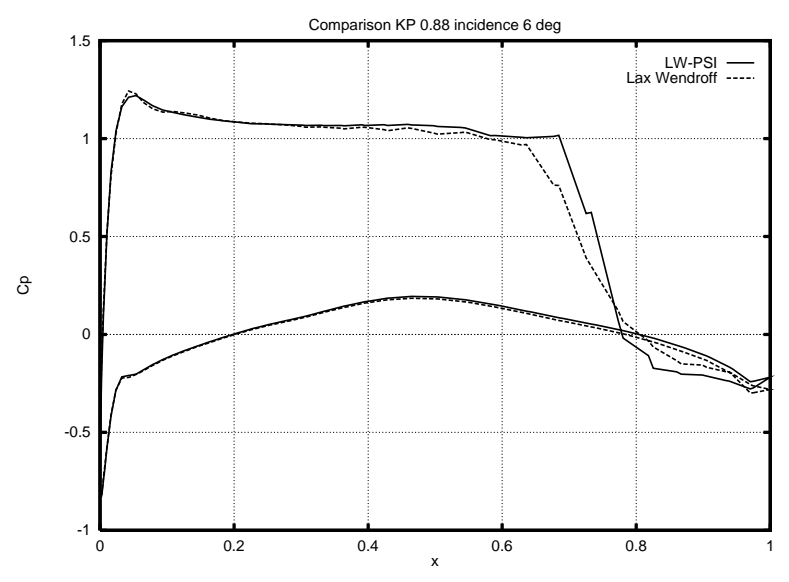

Figure 6. Comparison of $C_{p}$ along a profile.

The $\Sigma$ distributions (Fig. 7) show that entropy distributions are significantly more precise with the LW-PSI scheme than with the LX scheme. This accuracy is certainly provided by the formulation of the LW-PSI scheme because this formulation takes into account the conservation of the entropy along streamlines.

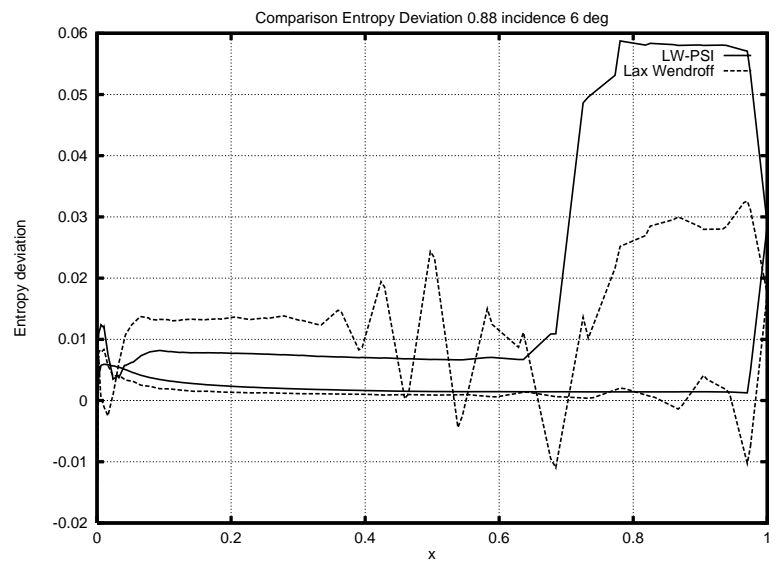

Figure 7. Comparison of $\Sigma$ along a profile.

We plot now the Mach number distributions along the same profile. 
The Mach number distributions (Fig. 8) show that the LW-PSI scheme has a less viscous behavior than the LX scheme since Mach numbers are more important for the LW-PSI scheme than for the LX scheme.

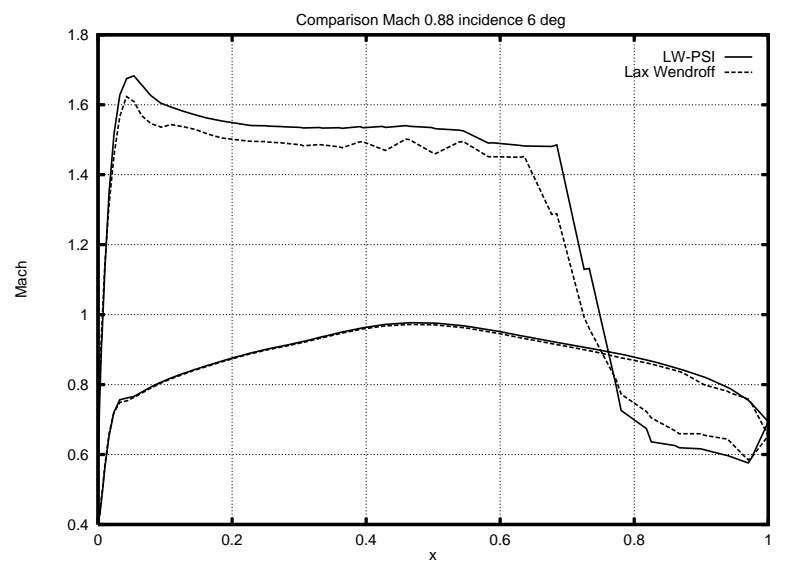

Figure 8. Comparison of Mach number along a profile.

We can notice that the shock wave is stronger for the LW-PSI scheme. The $C_{p}$ and Mach number distributions show no oscillations around the shock, but non physical spurious oscillations can be observed on the $\Sigma$ distribution of the LX scheme although the expectable behavior is obtained with the LW-PSI scheme.

The convergence history is plotted in Figure 9: we can notice the Newton type convergence starting around iteration 400 .

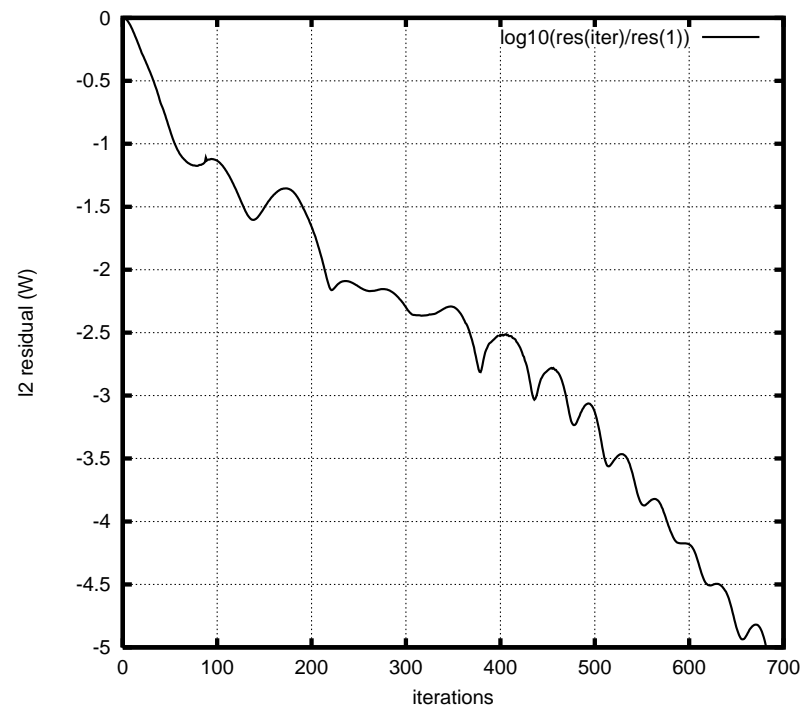

Figure 9. Convergence history for the transonic ONERA M6 wing test case.

\subsection{Low Mach cylinder}

In this test case, we compute a flow around a cylinder with a very low Mach number, $M_{\infty}=0.01$. Our aim is to show the ability of the LW-PSI scheme to compute nearly incompressible flows. 
We use a two dimensional mesh, with 11880 nodes and 23572 elements.

In Figure 10, we plot pressure iso-lines for the flows computed by the two different schemes (LW-PSI and LX). We compare these iso-lines with those of the analytical incompressible inviscid flow for the same Mach number. Indeed, for such a low Mach number flow, the solution is nearly incompressible.

This comparison (Fig. 10) show that the behavior of the LW-PSI scheme is quite good for low Mach number flows although the LX scheme has a poor behavior for such flows. This is likely due to the use of the van Leer-Lee-Roe matrix in the hyperbolic/elliptic decomposition.
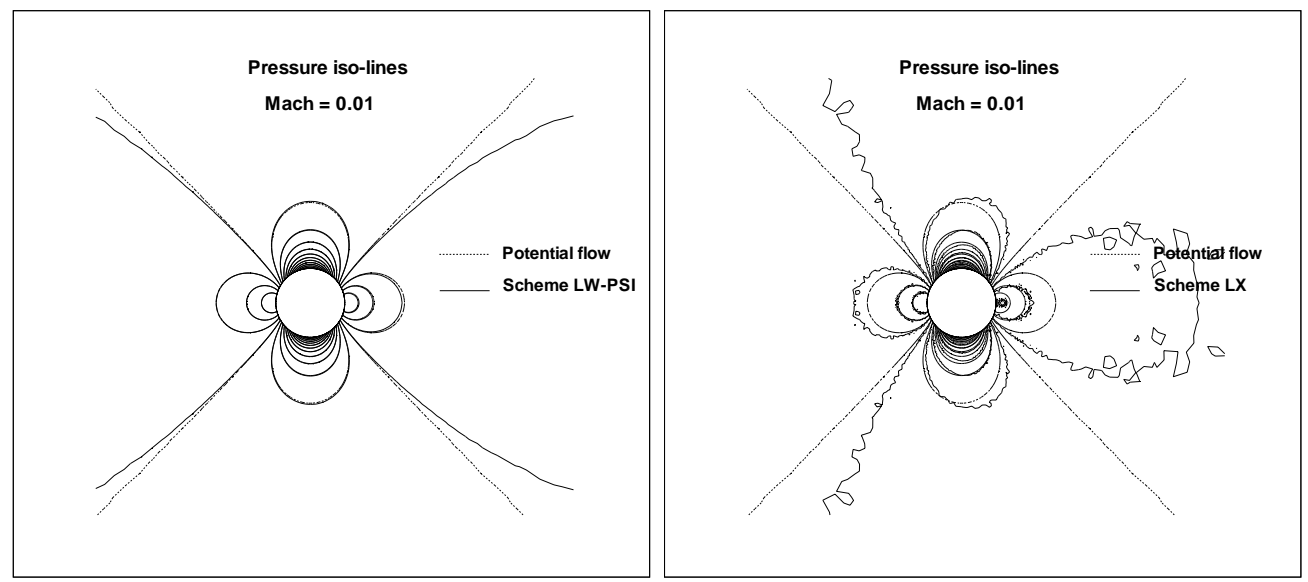

Figure 10. Pressure iso-lines for schemes LW-PSI (left) and LX (right).

Moreover, the two symmetric patterns, namely according to $x$-axis and $y$-axis, are captured by the LW-PSI flow solver.

\subsection{Subsonic zero-lift aircraft}

In this last test case, we compute the flow around a generic aircraft. We take $M_{\infty}=0.3$, in order to have a subsonic flow and thus no wave drag, and we select the incidence such that the lift is null and therefore the vortex drag. Thus, the total drag computed with this inviscid code should be zero.

We use a mesh with 45387 nodes and 255944 tetrahedra. The simulation includes the fuselage, wings, stabilizers, nacelles and pylones.

We plot the absolute entropy deviation over the aircraft (Fig. 11).

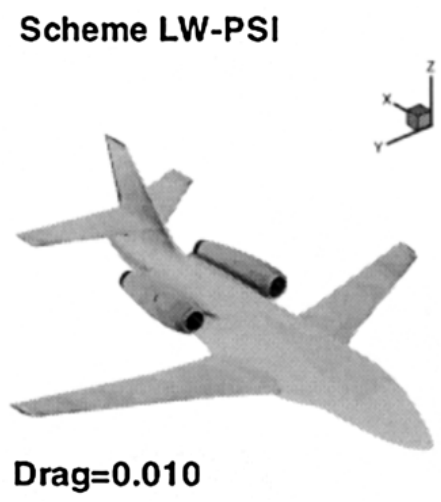

\section{Scheme LX}

\begin{tabular}{|l|}
\hline DEV \\
0.0015 \\
0.0014 \\
0.0013 \\
0.0012 \\
0.0011 \\
0.011 \\
0.0009 \\
0.0008 \\
0.0007 \\
0.006 \\
0.0005 \\
0.0004 \\
0.0003 \\
0.0002 \\
0.0001 \\
0 \\
\hline
\end{tabular}

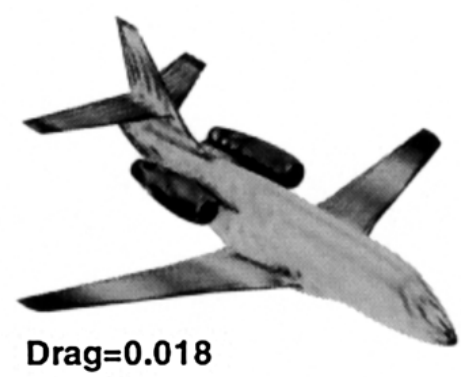

Figure 11. Absolute $\Sigma$ over a zero-lift aircraft. 
As in Section 6.1, we can confirm that the LW-PSI scheme generates much less entropy than the LX scheme. However, the computed drag is only $40 \%$ lower with the LW-PSI scheme than with the LX scheme. This is due to the poor quality of the mesh in the neighbourhood of the nacelles.

\section{COnCluding REMARKS}

A code has been developed which allows Euler computations over a large Mach number range since it is good for $M_{\infty}=0.01$ and we have made reliable three dimensional computations for $M_{\infty}=2$. In order to compute hypersonic flows, it will be necessary to extend this scheme to the Euler equations with chemistry, as done by Abgrall and Montagné [1] for the Osher scheme. The main issue is to know whether the additional equations for the chemical species must be included into the elliptic part or into the hyperbolic part of the decomposition.

The accuracy of the code allows precise drag computations and accurate shock resolutions, as it has been shown in Sections 6.1 and 6.4.

At this stage, we are satisfied of the additional viscous term (Sect. 4.3) we have added to the initial scheme of Paillère [9]. However we think that this term is not optimal because no theoretical study have been realised upon this term and thus the added viscosity may not be optimal.

This code is precise and robust enough to allow an industrial use although we have to acknowledge that this robustness and this accuracy is achieved at the expense of a higher cpu cost: at this step of the development of fluctuation splitting methods, we still have to make a choice between accuracy (LW-PSI scheme) and performances (LX scheme).

We would like to thank P.L. Roe, R. Abgrall, H. Paillère, H. Deconinck, E. van der Weide for their fruitful discussions on fluctuation splitting schemes and Q.V. Dinh, C. Sevin, T. Fanion for their collaboration in the implicitation of the scheme which is discussed in this paper. Special thanks are due to F. Chalot, M. Mallet, B. Stoufflet and M. Ravachol for their useful remarks and J. Francescatto for his advices. We thank the European Commission for supporting this work.

\section{REFERENCES}

[1] R. Abgrall and J.L. Montagné, Généralisation du schéma d'Osher pour le calcul d'écoulements de mélanges de gaz à concentrations variables et de gaz réels. La Recherche Aérospatiale No. 4 (1989) 1-13.

[2] V. Billey, Résolution des équations d'Euler par des méthodes d'éléments finis, application aux écoulements $3 D$ de l'aérodynamique. Ph.D. thesis, Université Pierre et Marie Curie (1984).

[3] A. Bonfiglioli, E. van der Weide and H. Deconinck, Study of 3D hypersonic flow using unstructured grid solvers based on multidimensional upwinding. von Karman Institute for Fluid Dynamics report (1996).

[4] H. Deconinck, Ch. Hirsch and J. Peuteman, Characteristic decomposition methods for the multidimensional Euler equations. Lect. Notes Phys. 264, Springer-Verlag (1986).

[5] H. Deconinck, H. Paillère, R. Struijs and P.L. Roe, Multidimensional upwind schemes based on fluctuation splitting for systems of conservation laws. J. Comput. Mech. 11 (1993) 323-340.

[6] H. Deconinck, P.L. Roe and R. Struijs, A multidimensional generalization of Roe's flux difference splitter for the Euler equations. J. Comput. and Fluids 22 (1993) 215-222.

[7] C. Faure and Y. Papegay, Odyssée Version 1.6 The User's Reference Manual. Rapport INRIA 0211 (1997).

[8] A. Jameson and W. Schmidt, Some recent developments in numerical methods for transonic flows. Comput. Methods Appl. Mech. Engrg. 51 (1985) 467-493, North Holland.

[9] H. Paillère, Multidimensional Upwind Residual Distribution Schemes for the Euler and Navier-Stokes Equations on Unstructured Grids. Ph.D. thesis, Université Libre de Bruxelles (1995).

[10] H. Paillère, H. Deconinck and E. van der Weide, Upwind residual distribution methods for compressible flow: an alternative to finite volume and finite element methods. von Karman Institute for Fluid Dynamics, presented at the 28th CFD Lecture Series (1997).

[11] J. Peraire, J. Peiro, L. Formaggia, K. Morgan and O.C. Zienkiewicz, Finite Element Euler Computations in Three Dimensions. Internat. J. Numer. Methods Engrg. 26 (1988) 2135-2159.

[12] P.L. Roe, Discrete models for the numerical analysis of time-dependent multidimensional gas dynamics. J. Comput. Phys. 63 (1986) 458-476.

[13] P.L. Roe, Linear advection schemes on triangular meshes. Technical report, Cranfield Institute of Technology, CoA 8720 (1987). 
[14] M. Rudgyard, Cell-vertex methods for steady inviscid flow. VKI LS 1993-04, Computational Fluid Dynamics (1993).

[15] Y. Saad and M.H. Schultz, GMRES: A Generalized Minimal Residual Algorithm for Solving Nonsymmetric Linear Systems. SIAM J. Sci. Statist. Comput. 7 (1986) 856-869.

[16] V. Selmin, A Multistage Method for the Solution of the Euler Equations on Unstructured Grids, in Proceedings of the fifth International Symposium on Numerical Methods in Engineering, Vol. 2, R. Gruber, J. Périaux and R.P. Shaw Eds., SpringerVerlag, Berlin, Heidelberg (1989) 449-454.

[17] D. Sidilkover, Multidimensional upwinding and multigrid, in 12th AIAA CFD Conference, San Diego, Paper 95-1759 (1995).

[18] D. Sidilkover and P.L. Roe, Unification of some advection schemes in two dimensions. Technical Report 95-10, ICASE (1995)

[19] D. Sidilkover, Numerical solution to steady-state problems with discontinuities. Ph.D. thesis, The Weizmann Institute of science, Rehovot, Israel (1989).

[20] R. Struijs, H. Deconinck, P. De Palma, P.L. Roe and K.G. Powell, Progress on multidimensional upwind Euler solvers for unstructured grids. AIAA-91-1550 (1991).

[21] R. Struijs, H. Deconinck and P.L. Roe, Fluctuation Splitting Schemes for the 2D Euler Equations. VKI LS 1991-01, Computational Fluid Dynamics (1991).

[22] B. van Leer, W.T. Lee and P. Roe, Characteristic Time-Stepping or Local Preconditionning of the Euler Equations, in AIAA 10th Computational Fluid Dynamics Conference (1991), AIAA-91-1552-CP. 